\title{
La physique de la turbulence Exposé de synthèse
}

\author{
The physics relating to turbulence \\ Synthesis report
}

\author{
M. Coantic
}

Professeur à l'Université d'Aix-Marseille II

Directeur de l'Institut de mécanique statistique de la turbulence

\begin{abstract}
L'exposé introductif rappelle tout d'abord les caractéristiques générales de la turbulence, phénomène à prévoir, mais aussi outil à utiliser. Puis sont présentées les idées récentes sur la transition et sa prévision, la dynamique de la turbulence homogène à deux ou trois dimensions, les structures cohérentes et leur rôle, l'organisation des zones à forts gradients et les progrès récents dans la description des couches limites turbulentes. Enfin, quelques interactions entre turbulence dynamique et autres processus physiques dans des situations complexes sont évoquées.
\end{abstract}

This introductory report first of all mentions the general characteristics of turbulence, a phenomenon to be expected, yet a tool which can also be made use of. Next are listed recent ideas concerning transition and its forecasting, the dynamics of homogeneous turbulence in two or three dimensions, coherent structures and their role, the organization of zones with steep gradients, and recent developments regarding the description of turbulent boundary layers. Finally, some interactions are listed occuring in complex situations between dynamic turbulence and other physical processes.

\section{Introduction}

Donner une définition précise de la turbulence des écoulements fluides reste encore aujourd'hui une tâche périlleuse.

Le passage d'un régime régulier à un régime chaotique, lorsqu'un paramètre caractéristique de l'importance des termes non linéaires dans les équations de Navier-Stokes dépasse un seuil critique, est un phénomène connu depuis plus d'un siècle. Les nombres de Reynolds très élevés rencontrés dans la plupart des applications industrielles font d'ailleurs que l'ingénieur traite en général des écoulements pleinement turbulents, encore que la détermination des zones de transition soulève parfois des problèmes. II est cependant des situations où la seule observation de l'irrégularité d'un écoulement, révélée par exemple par des visualisations, ne suffit pas à en garantir le caractère turbulent. Elle ne permettrait d'ailleurs pas de résoudre des problèmes comme celui de la détermination du point où s'achève effectivement la transition, ou encore celui de la possibilité de l'existence d'une turbulence bidimensionnelle.

Jusqu'à une date relativement récente, la définition généralement acceptée était que, pour qu'un écoulement soit turbulent, il faut qu'il présente effectivement l'ensemble des caractéristiques qui seront rappelées un peu plus loin. Depuis une quinzaine d'années, l'étude du comportement chaotique des «systèmes dynamiques » gouvernés par des équations différentielles non linéaires, a introduit des conceptions radicalement nouvelles sur les mécanismes d'apparition de la turbulence et les moyens de son identification. Compte-tenu des progrès réalisés dans cette voie, le temps n'est peut-être plus loin où l'ingénieur devra se familiariser à son tour avec les ésotériques « attracteurs étranges 》.

\section{LA HOUILLE BLANCHE/N 7/8-1987.}




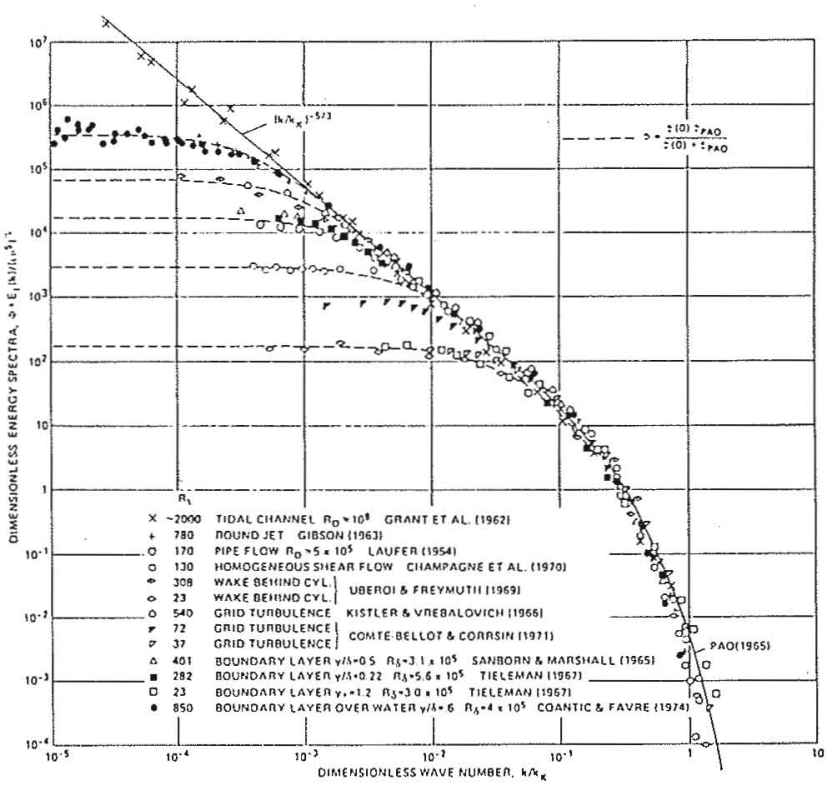

1. Représentation de spectres de divers écoulements urbulents rapportés aux échelles de Kolmogorov (d'après D.R. Chapman, 1979).

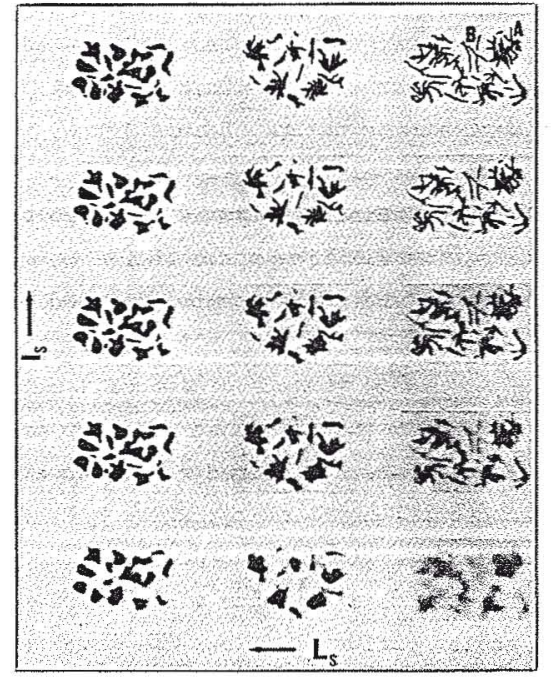

2. Le rôle de la turbulence dans l'homogénéisation d'un mélange (d'après Brodkey, 1975): dans ces schémas, la dispersion turbulente croit de gauche à droite et la diffusion moléculaire de haut en bas.
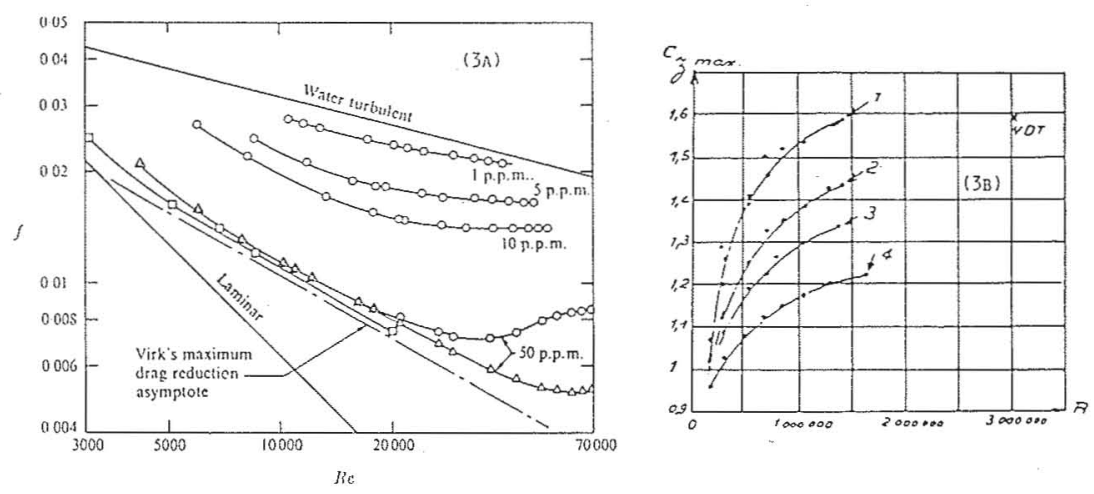

3. Quelques exemples du contrôle des effets bénéfiques ou néfastes de la diffusion turbulente:

a) réduction de perte de charge par addition de polymères (Paterson et Abernathy, 1972).

b) accroissement de la portance d'un profil par déclenchement de la turbulence (Rebuffet, 1950).

\section{Quelques caractéristiques générales de la turbulence}

\subsection{Le caractère aléatoire}

Les variations irrégulières et imprévisibles de la vitesse, et des autres variables attachées au fluide, en fonction de l'espace et du temps constituent la manifestation la plus immédiatement perceptible de la turbulence. Le recours à la méthode statistique est apparu très tôt comme nécessaire pour faire face au défi que représentaient ces variations et pour obtenir les propriétés moyennes nécessaires à l'ingénieur. En dépit du caractère un peu artificiel de la séparation du mouvement instantané en un "mouvement turbulent " et un "mouvement moyen " qui n'a en fait d'existence qu'au plan statistique, cette méthode est toujours la plus utilisée. L'étude expérimentale couplée à l'analyse théorique puis à la modélisation des divers types de moyennes en un point (à travers les "moments") ou en plusieurs points (à travers les "corrélations" et "fonctions spectrales») a en effet conduit à une bonne compréhension globale de la physique des écoulements turbulents et à des méthodes de prévision relativement fiables de leurs propriétés essentielles.

Cette approche classique est cependant aujourd'hui battue en brèche pour deux raisons. La première est une prise de conscience de plus en plus nette de ce que la turbulence n'est pas un phénomène totalement aléatoire puisque l'on peut y identifier des mouvements organisés, les "structures cohérentes", qui s'y répètent plus ou moins régulièrement et exercent une action parfois déterminante sur son évolution (cf. § III.3). La seconde est la possibilité offerte par les calculateurs les plus modernes d'une simulation numérique des irrégularités du comportement spatial et temporel des plus grandes échelles du mouvement turbulent, les "gros tourbillons », à d'ailleurs 
ne pas nécessairement confondre avec les "structures cohérentes " évoquées ci-dessus (cf. Thème n" 3 ). Ainsi, la frontière entre la partie de l'écoulement qui pourra être calculée directement (et qui devra l'être si l'on recherche une précision maximale), et celle qui relèvera d'un traitement purement statistique sera vraisemblablement dans quelques années fixée par l'ingénieur lui-même en fonction de critères de coût et d'efficacité.

Cette évolution n'est d'ailleurs pas sans poser des problèmes au niveau conceptuel : existe-t-il à la limite une "Physique de la turbulence" autre que celle contenue dans les équations de Navier?

\subsection{Le caractère non linéaire}

Une seconde caractéristique physique fondamentale de la turbulence est la coexistence en son sein d'une gamme large et continue d'échelles de mouvements. Ceci est une conséquence directe de la non linéarité des équations de Navier-Stokes : même si l'énergie est concentrée au départ dans un domaine spectral restreint, cette non linéarité a pour effet une répartition sur toute l'étendue disponible en un temps fini. La non linéarité assure par ailleurs une interaction permanente entre toutes les échelles. La prédicibilité des grosses structures est donc nécessairement limitée par l'effet des petites, dont réciproquement l'universalité du comportement statistique est compromise par l'influence des grosses.

La physique des interactions non linéaires est aujourd'hui assez bien appréhendée, tout au moins dans les situations les plus simples (cf. § III.2, et l'exposé de M. GenCE). Dans les cas pratiques de turbulence développée, les plus grosses structures, nécessairement inhomogènes et anisotropes en raison des conditions aux limites, possèdent les échelles maximales autorisées par ces dernières. Les "gros tourbillons", responsables des flux et de la production d'énergie cinétique du mouvement turbulent par interaction avec le mouvement moyen, à un taux noté $P$, ont des échelles typiques de vitesse et de dimension de l'ordre de $u^{\prime}=U / 5$ et $l^{\prime}=L / 5$ si $U$ et $L$ sont les échelles globales correspondantes de l'écoulement considéré. Pour la turbulence tridimensionnelle à laquelle est généralement confronté l'ingénieur, les interactions de ces gros tourbillons ont pour effet un transfert, ou "cascade", de l'énergie cinétique produite, à un taux de l'ordre de $u^{\prime 3} / l^{\prime}$, vers des échelles de plus en plus petites pour lesquelles le comportement local de la turbulence devient progressivement isotrope et homogène, et donc universel (cf. flg. l). Le processus n'est limité que par la viscosité, lorsqu'il atteint des échelles suffisamment petites pour que le flux d'énergie transféré soit dissipé en chaleur sous l'effet de celle-ci, à un taux noté $\varepsilon$. Les échelles des "petits tourbillons dissipatifs » sont celles de Kolmogorov, $v=(v \varepsilon)^{1 / 4}$ et $\eta=\left(v^{3} / \varepsilon\right)^{1 / 4}$, mais leur structure exacte reste mal connue (cf. § III.4). L'expérience et l'analyse des différentes constantes de temps montrent que la turbulence a tendance à être en "équilibre interne ", c'est-à-dire que les taux de production, de transfert, et enfin de dissipation sont, au moins en moyenne, approximativement égaux : $\varepsilon \cong P \cong U^{3} / 25 \mathrm{~L}$.

Ceci a des conséquences fondamentales pour des écoulements turbulents dont le nombre de Reynolds global, $R e=U . L / v$, est grand ou même très grand. La turbulence accroît la dissipation de l'énergie cinétique en chaleur dans une proportion considérable : son taux est en effet $\varepsilon \cong U^{3} / 25 L$ au lieu d'être de l'ordre de $v(U / L)^{2}$, soit un rapport de $R e / 25$ à 1 . Paradoxalement, cette dissipation accrue n'est pas contrôlée par la viscosité, qui n'agit que sur l'échelle à laquelle elle s'opère, mais par les échelles globales qui fixent les taux des mécanismes inertiels de production et de cascade. La gamme d'échelles mise en jeu est très étendue, puisque les échelles dissipatives sont de l'ordre de $R e^{-3 / 4}$ de la dimension des plus grosses structures. Enfin, les taux de cisaillement associés à ces petites échelles, de l'ordre de $\imath$ ' à celui, $U / L$, caractérisant le mouvement moyen $(v / \eta \cong$ $U / L \cdot R e^{1 / 2 / 5)}$.

Cet accroissement considérable des mécanismes moléculaires sous l'effet de la turbulence n'est pas limité à la dissipation visqueuse, mais s'étend aux diffusions de la chaleur ou de la masse dont l'efficacité crôt en fonction des cisaillements locaux. C'est bien pourquoi le recours au mélange turbulent s'impose chaque fois que l'on désire uniformiser la composition d'un mélange fluide. En l'absence de diffusion moléculaire, le brassage par la turbulence aurait déjà pour effet une dispersion des hétérogénéités initiales vers des échelles de plus en plus petites, avec un accroissement corrélatif de la surface de contact entre les composants (cf. fig. 2). Le mélange moléculaire s'exerce ainsi sur une surface considérablement accrue, mais son efficacité est encore augmentée par les cisaillements locaux qui réalisent un "flux de variance " vers les plus petites échelles, assez analogue à la cascade de l'énergie cinétique.

\subsection{Le caractère diffusif}

L'effet de la turbulence qui a certainement la plus grande importance pratique pour l'ingénieur est sa capacité à diffuser spatialement toutes les grandeurs attachées au fluide avec une efficacité très largement supérieure à celle de la diffusion moléculaire. Par exemple, pour la quantité de mouvement, le rapport de la "viscosité turbulente" à la viscosité moléculaire est de l'ordre de $v_{i} / v \cong u^{\prime} l^{\prime} / v \cong U L / 25 v=R e / 25$ (on retrouve naturellement le rapport des taux de dissipation).

Les causes et les effets de cet accroissement des transferts au sein du fluide sont trop bien connus pour que nous nous y attardions. Un point sur lequel il convient cependant d'insister est que la diffusivité turbulente, étant une caractéristique non pas du fluide mais de l'écoulement, peut être dans une plus ou moins grande mesure contrôlée en agissant sur ce dernier. Lorsque, comme c'est souvent le cas en aéro ou en hydrodynamique, la diffusion turbulente a des effets néfastes d'accroissement des trainées de frottement ou des pertes de charge, on s'efforce d'en retarder l'apparition ou d'en diminuer le niveau (cf. l'exposé de M. Coustelx). Il est cependant bien des situations où la diffusion turbulente a des effets bénéfiques : on s'efforce alors de la favoriser (cf. fig. 3). C'est la situation de loin la plus fréquente dans les génies ther'mique et chimique où l'on recherche des transferts de chaleur et de matière les plus élevés possibles (cf. l'exposé de M. Burnage). 

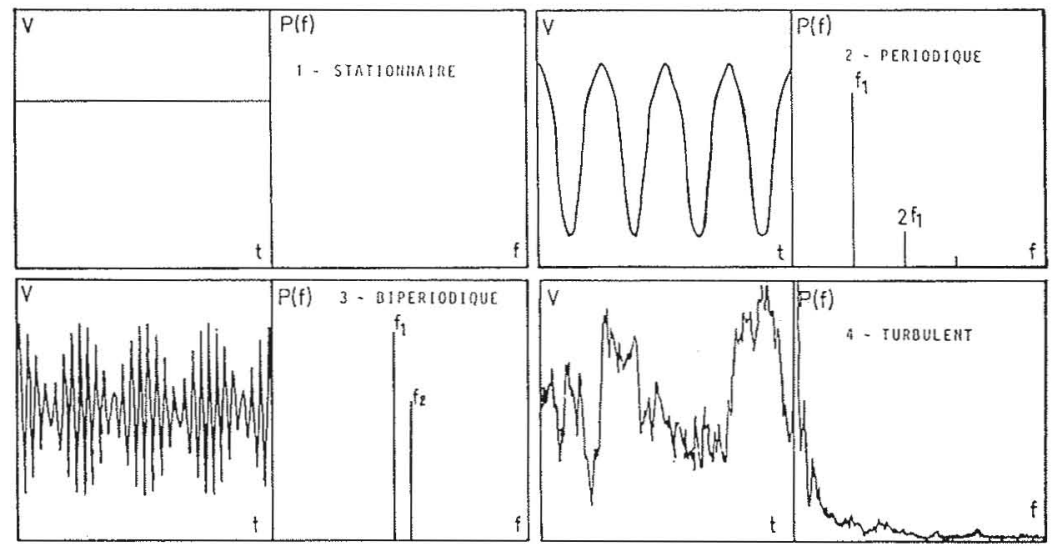

4. La succession d'états conduisant à la turbulence pour l'instabilité de Rayleigh-Bénard dans une boite de petit allongement d'après Bergé et Pomeau (1980).

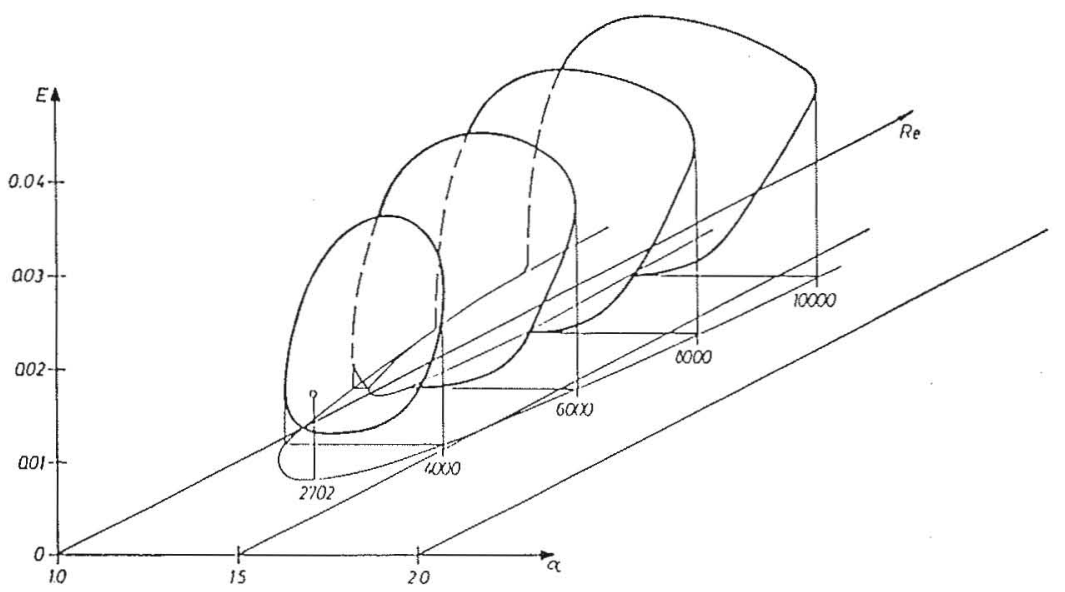

6. Surface de stabilité neutre pour l'écoulement de Poisenille entre plaques parallèles (Hilbert, 1977): la transition est possible pour $2702 \leqslant R \leqslant R_{r} \cong 5600$.

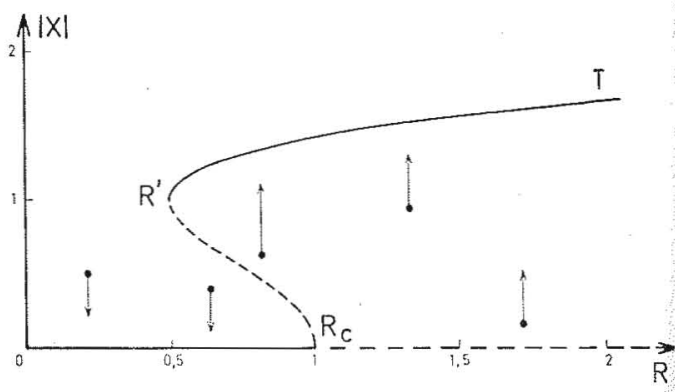

5. Exemple de bifurcation souscritique.

Le système $d x / d T=x\left(R-\left(X^{4} / 2\right)-X^{2}+I\right)$ possède des solutions stationnaires sur l'ensemble des courbes out son second membre s'annule. Mais des branches sont stables (et d'autres instables (---). Pour $I / 2 \leqslant R \leqslant R_{i}=1$, on bifurque d'une solution d'amplitude nulle $O R_{c}$ vers une solution d'am. plitude finie $R$ 'T lorsque la perturbation initiale dépasse la courbe $R^{\prime} R_{t}$.
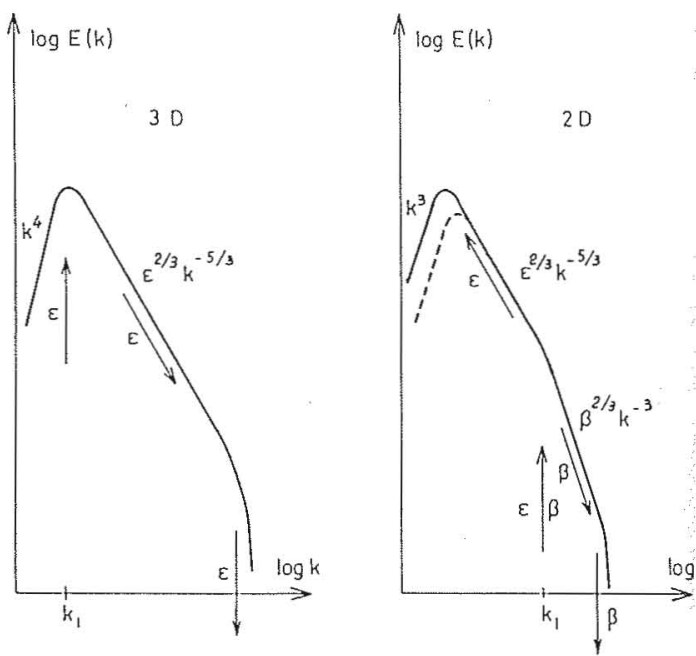

7. La dynamique spectrale de la turbulence à trois et deux dimensions pour des injections d'énergie, $1 / 2 v_{i}^{\prime} v_{i}^{\prime}$, et d'enstros phie $1 / 2 \omega^{\prime} \omega^{\prime}$, à des taux constant $\theta$ et $\beta \cong k_{i}^{2} \theta$, et des échelles voisines de $1 / k_{i}$.
La diffusion par la turbulence est très généralement modélisée par l'intermédiaire de « coefficients de diffusivité turbulente». Les limites de cette approche sont aujourd'hui bien connues : la dispersion par la turbulence met en jeu des échelles de plus en plus grandes à mesure que l'on s'éloigne de la "source", et, dans les situations permanentes, fait donc intervenir les échelles les plus grandes présentes dans l'écoulement. Ces dernières devraient rester petites vis-à-vis des échelles de variation des variables moyennes considérées pour que le concept de diffusivité turbulente, impliquant une relation de proportionnalité entre des flux et des gradients locaux, ait un sens. Cela étant impossible, ce concept se trouvera plus ou moins gravement en défaut dès que l'on sortira des situations les plus simples: un exemple bien connu est l'apparition de flux à contre-gradient.

En conclusion, la physique de la turbulence intéresse l'ingénieur à deux titres. D'abord parce que la turbulence est un phénomène auquel il est très fréquemment confronté et qu'il doit donc bien connaître pour mieux en comprendre, et si possible en prévoir les effets. Ensuite parce que la turbulence apparaît de plus en plus comme un outil ( $c$. thème $\mathrm{n}^{\circ} 4$ ) dort il doit pouvoir mieux maîtriser l'emploi grâce à une meilleure connaissance de son origine et de ses mécanismes. 


\section{Quelques développements récents}

\subsection{Nature du chaos turbulent, et mécanisme de la transition}

Une véritable révolution conceptuelle s'est opérée ici avec l'introduction de la notion de "chaos déterministe", la démonstration de la possibilité de divers types de «bifurcations " et enfin l'étude des diverses "routes vers le chaos". Nous en donnons quelques idées élémentaires, renvoyant pour le reste à des travaux spécialisés ou de vulgarisation (Bergé et Pomeau, 1980; Swinney et Gollub, 1981; Lanford, 1982; Bergé et al., 1984; Guckenheimer, 1986; Dubois et al., 1987).

Le fait essentiel est que le comportement désordonné caractéristique de la turbulence n'est pas nécessairement lié à la présence d'un très grand nombre de perturbations indépendantes (les «degrés de liberté » introduits par Landau en 1944) mais peut s'observer dans des systèmes mécaniques non linéaires mais parfaitement déterministes mettant en jeu un minimum de trois périodes différentes (ceci a été montré par Lorenz en 1963 et Ruelle et Takens en 1971). L'évolution d'un tel système est parfaitement déterminable, et l'on montre qu'il tend dans l'« espace des phases » (où un point représente l'ensemble des variables nécessaires pour caractériser complètement l'état du système à un instant donné) vers un "attracteur étrange " constituant un « objet fractal». Cette prédicibilité théorique est cependant sans aucun sens pratique, des écarts infinitésimaux sur l'état initial conduisant à des évolutions totalement dissemblables du système, dont les « trajectoires " divergent exponentiellement: c'est la "sensibilité aux conditions initiales ».

La transition du régime régulier au régime chaotique s'effectue lorsqu'un "paramètre de contrôle " du système (par exemple un nombre de Reynolds) croit, mais suivant des «scénarios» variés. La succession des régimes est bien comprise dans certains systèmes fluides à petit nombre de degrés de liberté (instabilité convective de Rayleigh-Bénard dans une petite boîte ou instabilité de Taylor-Couette entre cylindres concentriques) comme l'illustre la figure 4. Dans d'autres systèmes, on observe des "bouffées" irrégulières de turbulence, ou des doublements successifs de périodes ou encore de la «turbulence de phase ".

Le passage d'un régime à un autre constitue une "bifurcation" de la solution des équations du système : celle qui est suivie devient instable et l'on "bifurque" vers une autre, généralement plus compliquée. Mais il faut ici tenir compte de l'amplitude des perturbations présentes dans le système, ce qui conduit à distinguer des instabilités "supercritiques" ou "souscritiques ». Pour les premières, la bifurcation est indépendante de l'amplitude des perturbations et ne peut apparaître que pour des valeurs du paramètre de contrôle, $R$, égales ou supérieures à la valeur " critique ", $R_{\varepsilon}$, déterminée pour une amplitude infinitésimale. Pour les secondes, la bifurcation est possible pour des valeurs de $R$ inférieures à $R_{i}$ si l'amplitude est suffisante, comme l'illustre la figure 5 .

Ces diverses considérations apportent une explication cohérente à la variété des mécanismes de la transition à la turbulence des écoulements fluides, et à leur dépendance des perturbations initiales. On observera tantôt une complexification progressive de l'écoulement, tantôt un passage brutal et intermittent à un régime fortement perturbé. Les cas de bifurcations souscritiques sont fréquents et importants (cf. fig. 6) : par exemple, la transition dans le tube circulaire, où le nombre de Reynolds critique de l'écoulement de Poiseuille pour une perturbation infinitésimale est infini !

Le problème, très important en pratique, de la transition de la couche limite faiblement perturbée conserve son intérêt à l'approche linéarisée: il semble en effet que la première bifurcation corresponde à l'apparition des modes bidimensionnels du type Tollmien-Schlichting, le développement ultérieur des "spots de turbulence" tridimensionnels étant supercritique. Les progrès récents (cf. Reshotko, 1976; Swinney et Gollub, 1981) montrent l'importance du non-parallélisme ou encore du transfert de chaleur: pour un écoulement d'eau, une élévation de température de la paroi de 2,8 " $\mathrm{C}$ seulement entraînerait un doublement de la distance du bord d'attaque au point critique.

La turbulence développée à grand Reynolds possède un grand nombre de degrés de liberté, et la validité des approches précédentes y est encore discutable : d'après des travaux récents (Aubry et al., 1987), les structures cohérentes dont il sera question plus loin pourraient s'y comporter comme des systèmes dynamiques relativement simples.

\subsection{Dynamique de la turbulence en deux et trois dimensions}

La théorie statistique de la turbulence homogène et isotrope est vieille d'un demi-siècle. Cependant, le problème fondamental du calcul du taux de transfert spectral d'énergie dans l'équation d'évolution du spectre obtenue à partir de l'équation de Navier n'a été résolu jusque vers la fin des années 50 que par des modélisations globales simples. Des théories beaucoup plus élaborées ont depuis été mises au point par Kraichnan, puis aux Etats-Unis des auteurs comme Orszag, et en France toute une école (ne pouvant rendre ici justice à tous, je citerai seulement $\mathrm{U}$. Frisch et M. Lesieur).

On somme ici explicitement l'ensemble des interactions non linéaires entre "triades de vecteurs d'ondes " conduisant à des "cascades" directes et inverses d'énergie et d'enstrophie dans le domaine spectral. Dans la théorie "E.D.Q.N.M. ", largement utilisée ces dernières années, les interactions sont évaluées à partir d'une hypothèse de quasi-normalité des moments quadruples corrigée, pour des raisons physiques, par des hypothèses de "Markovianisation " puis d' « amortissement turbulent " (cf. Lesieur, 1978, 1983; André et Barrère, 1981).

Ces travaux ont conduit à une compréhension beaucoup plus fine de la dynamique interne des divers types de turbulence (cf. fig. 7) et de leurs évolutions spectrales et globales. Certains de leurs résultats, par exemple sur les questions de "prédicibilité " ou de viscosité turbulente spectrale (cf. Chollet, 1982), sont directement utiles à l'ingénieur. Encore plus intéressantes pour lui sont les théories de la turbulence homogène anisotrope, développées en particulier par des chercheurs lyonnais, où l'interaction entre les champs moyen et turbulent est prise en considération, et dont traitera M. Gence. 

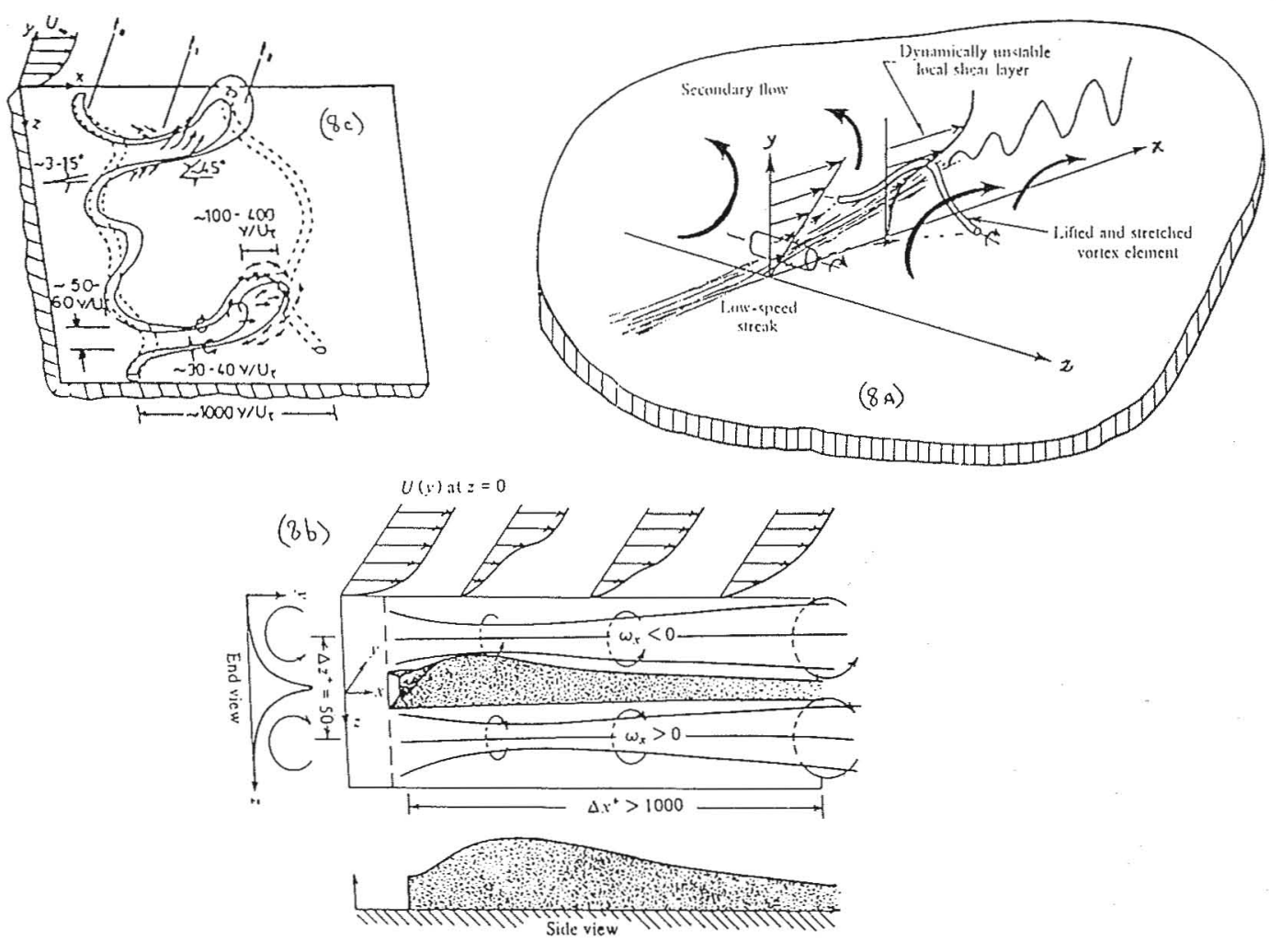

8. Schémas de la structure de la turbulence près d'une paroi d'après:

(a) Kline et al. (1967)

(b) Blackwelder et al. (1979)

(c) Wallace (1985)

\subsection{Divers types de structures cohérentes}

L'identification de mouvements relativement bien organisés au sein de certains types d'écoulements turbulents est l'un des évènements marquants des dernières années. Elle repose sur des visualisations et des techniques d'analyse du signal ou de reconnaissance de formes plus ou moins évoluées (Cantwell, 1981; Lumley, 1981; Hussain, 1983 $a, b)$. Hussain définit une structure cohérente comme une masse simplement connexe et à grande échelle de fluide turbulent possédant une vorticité corrélée en phase spatialement, ou «vorticité cohérente ». Pas nécessairement très énergétiques, ces mouvements cohérents jouent un rôle significatif dans les transports turbulents mais sont baignés dans une turbulence « incohérente » dont l'effet peut également être important.

Pour les écoulements de paroi, c'est Kline et ses collègues qui ont été les premiers à mettre clairement en évidence un mécanisme désormais classique (cf. fig. 8): apparition de "raies " alternativement lentes et rapides associées à des tourbillons longitudinaux contra-rotatifs; élévation, instabilité puis "explosion» (bursting) des zones lentes; enfin «balayage" (sweep) par une masse de fluide rapide et reprise du processus. La cinématique de ces structures de paroi est aujourd'hui bien connue, mais leur dynamique et leurs liens avec les structures de la zone externe restent à mieux élucider. Ces dernières structures sont d'ailleurs mal identifiées, sauf peut-être à l'interface turbulent-laminaire où les " bosses " turbulentes montrent sur leur face aval un point de stagnation relative (cf. fig. 9) présentant une certaine analogie avec celui observé dans les écoulements libres. Le comportement de ceux-ci, dont le prototype est la couche de mélange étudiée notamment par Roshko puis Hussain, apparaît comme dominé par de gros tourbillons transversaux reliés entre eux par des nappes de fins tourbillons longitudinaux qui sont en 


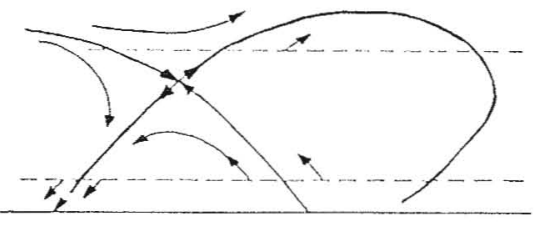

9. Schéma de la structure de la zone externe de la couche limite turbulente pour un observateur se déplaçant à $0,8 U_{\infty}$ (Falco, 1977).

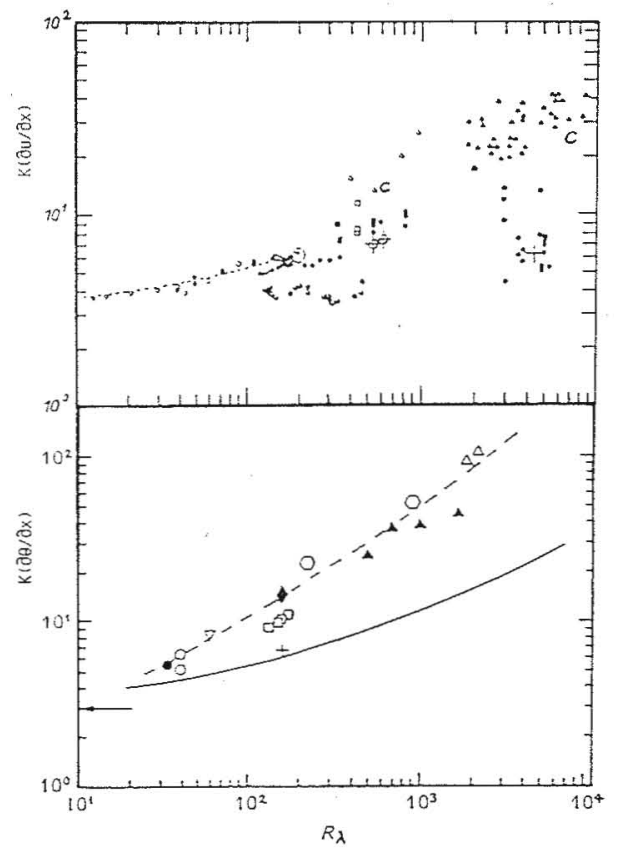

11. Variation d'un indicateur de l'intermittence, le facteur d'aplatissement des dérivées longitudinales de la vitesse et de la rempérature, en fonction du nombre de Revnolds de la turbulence, $R \lambda$, dans divers écoulements (d'après Mestayer, 1982, et Tavoularis et Corrsin, 1981).
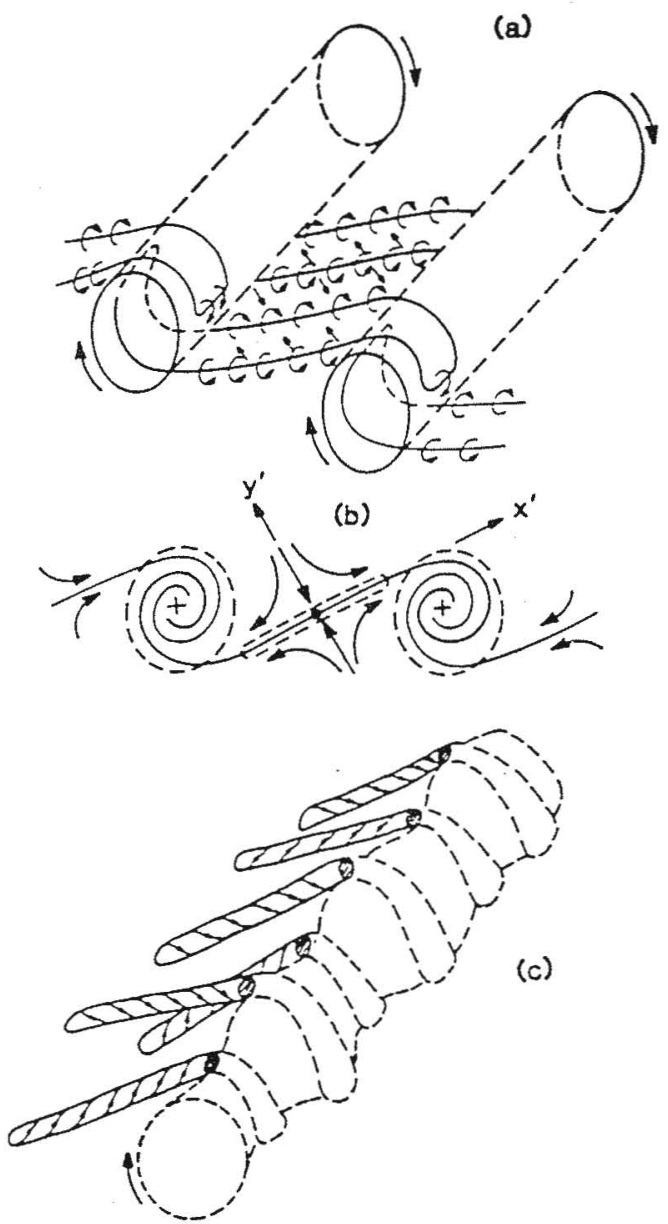

10. Schéma de la structure de la couche de mélange d'après Hussain (1983): les nappes de petits tourbillons raccordant les gros $(a)$; vue latérale $(b)$; détail du raccordement (c). constante extension et alimentent en énergie turbulente les gros (cf. fig. 10) : il semble s'agir là du cas où les structures cohérentes sont les plus importantes et organisées.

Deux écoles existent, comme l'a noté Lumley: pour l'une, les structures cohérentes sont toujours présentes et importantes; pour l'autre, elles sont caractéristiques d'une turbulence "jeune » et destinées à disparaitre au fur et à mesure de son développement. Sans vouloir trancher, nous soulignerons ici deux points :

- L'existence de structures cohérentes explique souvent les phénomènes de diffusion à contre-gradient, ou production négative : leur prise en compte explicite est alors utile, ou peut être même nécessaire, à la modélisation.

- Ces structures peuvent rester très longtemps affectées par les conditions de leur création : ceci ouvre la voie à des possibilités de modification artificielle des propriétés de la turbulence par une excitation locale judicieusement appliquée : on cite des résultats spectaculaires.

\subsection{Organisation des zones dissipatives}

L'organisation spatiale et temporelle des échelles les plus fines de la turbulence contrôle par exemple certains phénomènes de combustion ou encore de propagation de rayonnements. Le problème est de savoir si elle possède effectivement le caractère universel supposé implicitement par la théorie de Kolmogorov de 1941.

La nature intermittente des structures fines est un fait aujourd'hui bien connu: le pourcentage du volume turbulent qu'elles occupent décroît lorsque le nombre de Reynolds augmente (cf. fig. 11 ). Divers modèles de fractionnement autosimilaire ont été proposés pour expliquer ce phénomène. Les résultats d'Anselmet et al. (1984) sont plutôt en faveur du modèle lognormal de Yaglom que du $\beta$-modèle de Frisch et al. Le paramètre d'intermittence $\mu=3-D$ où $D$ est la dimension fractale des zones dissipatives serait égal à $0,2 \pm 0,05$. 

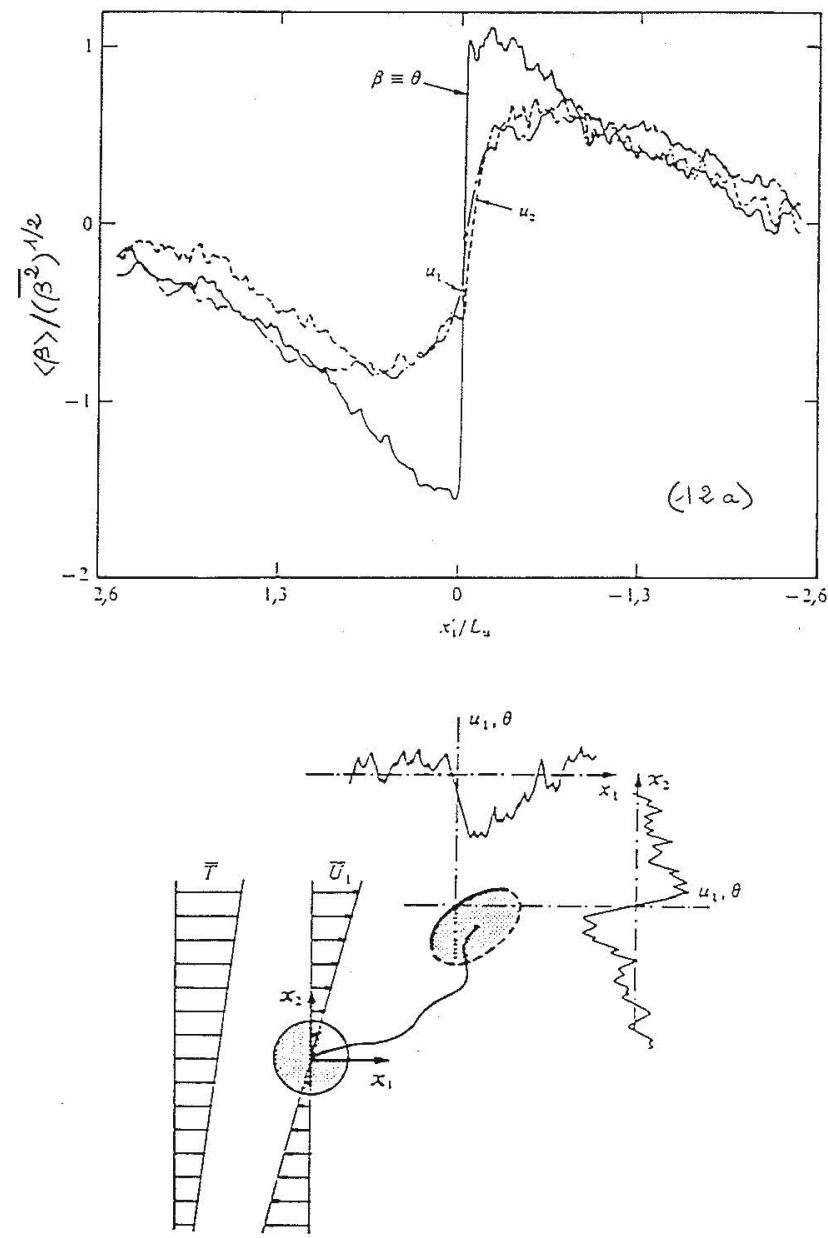

12. La présence de "fronts internes" au sein des écoulements turbulents cisaillés :

(a) moyenne conditionnelle du front thermique et dynamique dans un jet, d'après Antonia et al. (1986).

(b) schéma qualitanif général de Tavoularis et Corrsin (1981).

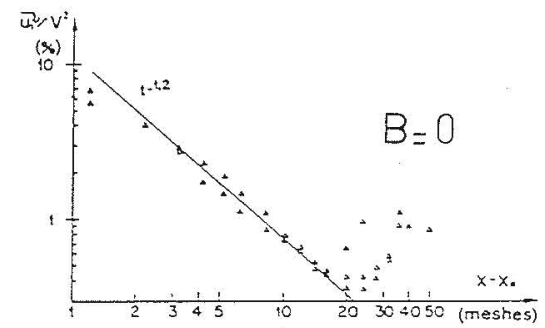

(a)

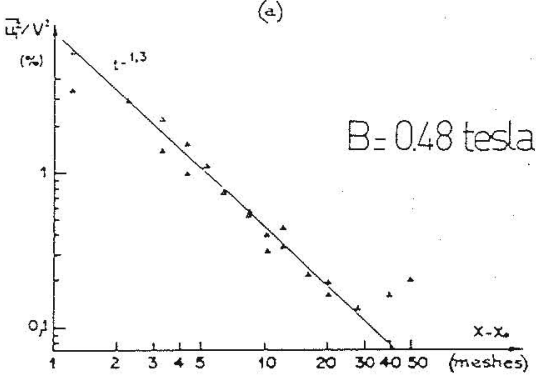

(b)

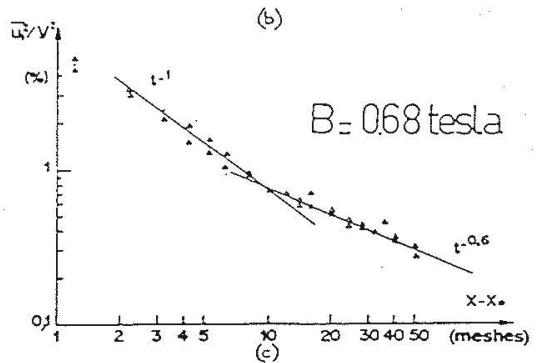

15. Evolution d'une turbulence de grille M.H.D. pour différents niveaux du champ magnétique, d'après J. Sommeria (1983).

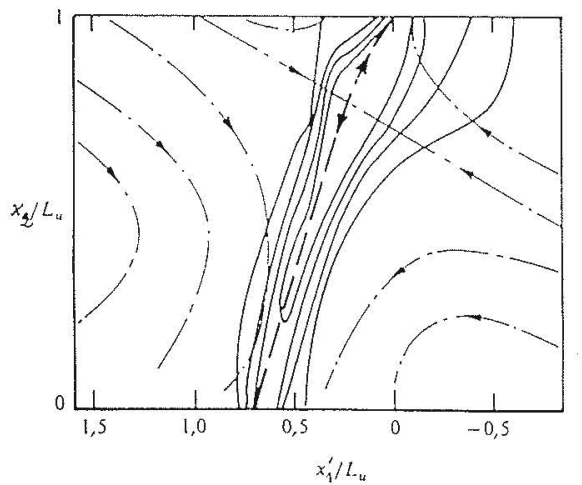

13. Relation entre le niveau de "dissipation" de la variance de température et la position du "front thermique " en extension d'après Antonia et al. (1986).
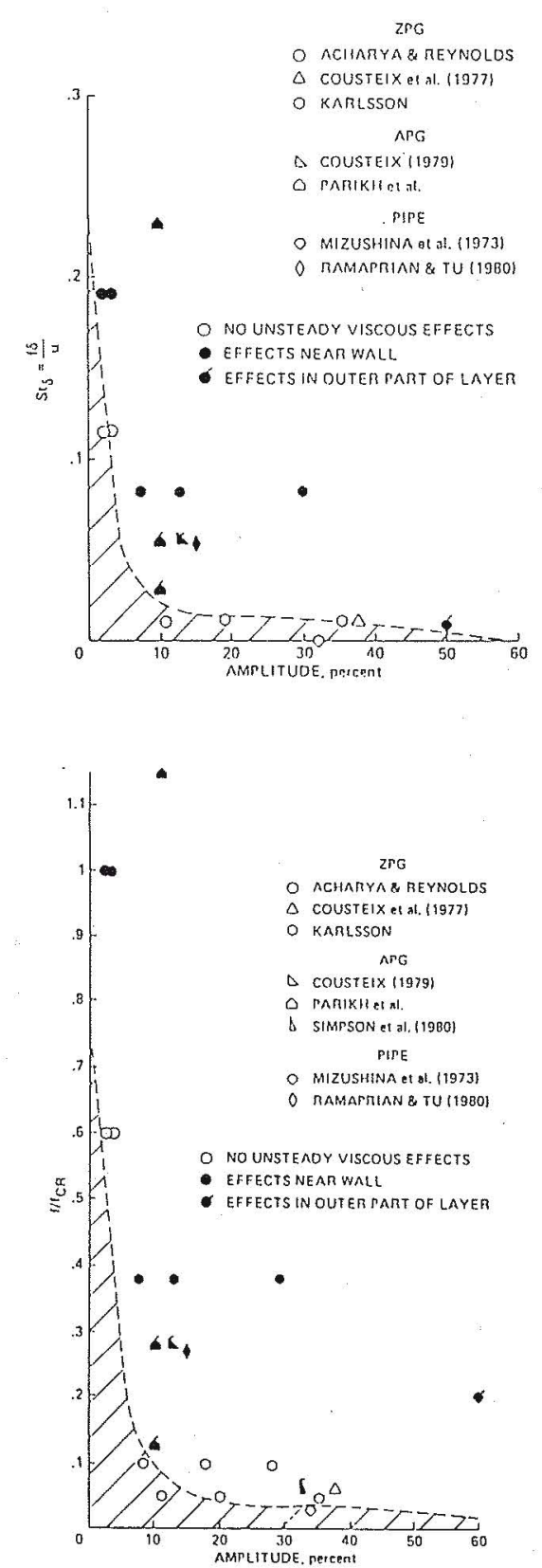

14. Importance des effets instationnaires d'après Carr (1981): l'ordonnée est le nombre de Strouhal (a) ou le rapport de la fréquence à la fréquence de brusting (b). 
Savoir si ces échelles sont ou non localement isotropes constitue une question importante et controversée. Des travaux récents (voir Gibson et al., 1977; Tavoularis et Corrsin, 1981; Mestayer, 1982; Antonia et al., 1986) aboutissent à des conclusions plutôt contradictoires suivant les critères adoptés pour en juger. Si l'on se fie aux corrélations et fonctions de structure, l'isotropie est plutôt bien vérifiée, et mieux encore pour la vitesse que pour la température. Cependant, elle ne l'est guère si l'on compare directement divers termes de dissipation ou si l'on tient compte de la non-nullité des moments impairs de certaines dérivées. En particulier, l'étude du comportement du coefficient de dissymétrie de la dérivée longitudinale de la température $\left.S=\overline{\left(\partial \theta^{\prime} / \partial x\right.}\right)^{3} /\left(\overline{\left(\partial \theta^{\prime} / \partial x\right)^{2}}\right)^{3}$, a mis en évidence l'effet des structures à grande échelle, nécessairement anisotropes, sur des paramètres supposés a priori déterminés par les seules échelles dissipatives, et donc isotropes: les résultats s'expliquent par la présence de "fronts internes", clairement identifiés dans certains cas et dont la présence est vraisemblable dans tous les écoulements cisaillés (cf. fig. 12).

On peut enfin s'interroger sur la géométrie des zones dissipatives et leur localisation éventuelle vis-à-vis des structures cohérentes. Des indications expérimentales, et des considérations sur l'extension des filets tourbillonnaires, leur donneraient plutôt l'allure de filaments cylindriques essentiellement concentrés dans les zones d'allongement par les grosses structures (cf. fig. 13). On notera, mais avec beaucoup de prudence, l'analogie d'un tel schéma, dont la généralité reste d'ailleurs à démontrer, avec celui de la figure 10 .

\subsection{Progrès dans la description des couches limites turbulentes}

On ne tentera pas de synthétiser ici le flot continu des travaux sur cet important sujet : pour des revues récentes, consulter Bradshaw, 1978; Cebeci et Smith, 1974; les proceedings des Conférences de Stanford de 1968 et 1981 tot de Symposia IUTAM, AGARD et T.S.F., la collection des Annual Reviews ou Fluid Mechanics.

La structure des couches limites «simples» étant désormais bien comprise et modélisée, on attaque des problèmes plus difficiles, comme les situations instationnaires périodiques et les tridimensionnelles (cf. Michel et al., 1981; Cousteix, 1986). Pour les premières, les profils de vitesse moyennés sont souvent voisins de ceux qui correspondraient à la situation moyenne, et la structure urbulente cyclique quasi-stationnaire. Les effets instafionnaires sont fréquemment confinés à la proximité de la paroi. lls sont plus marqués si l'amplitude et la fréquence des oscillations sont plus grandes et le gradient de pression est défavorable, mais sans liaison nette avec le rapport de la fréquence imposée à celle du "bursting » de la sous-couche (fig. 14). La prévision du frottement des couches limites minces tridimensionnelles est encore peu antisfaisante, ce qui traduit des écarts de structure de la urbulence vis-à-vis des situations classiques d'équilibre. L'expérience montre en particulier que la viscosité turbutonte près de la paroi est loin d'être la même dans les différentes directions (Arzoumanian et al., 1981).
Dans un cas comme dans l'autre, le problème le moins avancé reste la prévision du décollement (voir par exemple AGARD C.P. 386, 1985; Smith, 1986). Les données expérimentales sont rares et difficiles à obtenir. La compréhension physique est compliquée, d'une part par le caractère fondamentalement instationnaire, même en situation stationnaire, du phénomène, d'autre part, par l'importance du couplage de la couche limite proche du décollement avec la zone non visqueuse de l'écoulement et la zone tourbillonnaire détachée.

Mentionnons pour finir un vif renouveau d'intérêt pour les méthodes de " manipulation» de la couche limite dont traitera M. Cousteix.

\subsection{Quelques situations turbulentes complexes}

La turbulence purement dynamique d'un fluide monophasique homogène et isotherme soumis à la seule pesanteur constitue presque une exception pour l'ingénieur, fréquemment confronté à des situations plus complexes. Nous évoquerons ici quelques cas où les processus en interaction avec la turbulence n'en subissent pas seulement l'effet mais en modifient la structure.

Les effets gravitationnels des fluctuations de densité souvent associées aux transferts de chaleur et de masse sont les mieux connus en raison de leur immense importance pratique. La turbulence bien développée dans les zones faiblement stratifiées et les régions instables est relativement bien comprise et modélisée (même lorsqu'elle est le siège de flux à contre-gradient). Il n'en est pas de même de la turbulence affaiblie et souvent instationnaire des zones stables, et des problèmes comme l'érosion des thermoclines ou la dégénérescence en ondes internes d'une turbulence 3D sont l'objet d'actives recherches (cf. Métais, 1985).

Une rotation rapide, ou l'action d'un champ magnétique en MHD, ont pour effet une bidimensionnalisation de la turbulence dont la dynamique et l'évolution peuvent être profondément modifiées (fig. 15).

Dans les combustions, transferts de chaleur et de masse et turbulence dynamique sont intimement liés. En effet, les taux locaux de réaction dépendent non seulement des concentrations mais encore, très fortement, de la température dont l'évolution résulte au premier chef des quantités de chaleur dégagées (Libby et Williams, 1980). De tels couplages suffisent par eux-mêmes à provoquer un comportement turbulent du système (instabilité des «flammes laminaires "). En régime turbulent, les possibilités sont nombreuses : fronts de flamme « froissés " par les tourbillons pour les combustions lentes avec prémélange; géométrie de flamme complexe liée à celle de la surface de mise en contact des réactifs pour les combustions rapides sans prémélange; etc. L'intermittence spatiale et temporelle est d'ailleurs une caractéristique commune à tous les processus physiques, et même biologiques, associés à la turbulence: que l'on songe à la forme des nuages ou au «spottiness " de la répartition du plancton dans l'océan!

Les écoulements turbulents polyphasiques soulèvent toute une gamme de problèmes nouveaux. Nous ne citerons ici que celui des écoulements chargés en particules, sur lequel reviendra $\mathrm{M}$. Burnage. La présence au sein d'une turbulence cisaillée de particules possédant des 
densités et des vitesses nécessairement différentes de celles du fluide adjacent a des effets variés. Pour des particules lourdes, on va observer des effets de stratification stable tout à fait analogues à ceux mentionnés plus haut. Même pour des particules légères, les échelles dissipatives sont accrues, ce qui entraînera une augmentation de l'épaisseur des sous-couches visqueuses et donc une réduction du frottement (Lumley, 1978).

\section{Conclusion}

Quel sens peut-on attacher aujourd'hui à l'expression "Physique de la turbulence" ? Si l'on admet au départ, comme il y a de bonnes raisons de le faire, que tous les détails d'un écoulement turbulent obéissent à la physique que décrivent les équations de Navier, il est évident que la question doit être précisée.

On comprend bien à présent pourquoi, et l'on commence à savoir comment, un système d'équations déterministe peut conduire à des solutions chaotiques. L'existence de la turbulence est donc un problème physique résolu, au moins au niveau conceptuel.

Il reste alors à savoir si des lois particulières peuvent être mises en évidence dans son comportement. Une première approche du problème est, en quelque sorte, interne: elle consiste à rechercher au sein du chaos turbulent des éléments constitutifs identifiables possédant si possible un caractère universel. C'est ce à quoi s'efforcent les études de «structures» évoquées plus haut. S'il est possible d'identifier des éléments constitutifs jouant un rôle suffisamment important et général, et d'élucider les lois gouvernant les interactions de ces éléments, on pourra évidemment remonter à une "physique " globale de la turbulence.

L'autre approche, que nous qualifierons d'externe, part d'un point de vue plus statistique et global : elle consiste à rechercher les lois de comportement du «matériau turbulent " soumis à différentes sollicitations. Les échelles de temps et d'espace sur lesquelles s'effectue l'analyse jouent ici un rôle déterminant. Ainsi, les classiques mesures de moyennes temporelles «en un point» ont permis d'établir des relations constitutives entre les moments d'un certain ordre et le champ des divers moments d'ordres inférieurs dans le voisinage du point considéré, la notion de voisinage se bornant souvent à la considération de dérivées premières. Inconvénient bien connu : cette procédure conduit à des relations de proportionnalité entre des flux et des gradients locaux, en contradiction avec le caractère non local des transferts opérés par les "gros tourbillons". Le recours au filtrage associé à l'approche spectrale de la turbulence permet de pallier en partie à cet inconvénient, en limitant les lois de comportement recherchées à des échelles assez petites pour que l'on puisse escompter qu'elles dépendent seulement des gradients locaux. Il est manifeste ici que le concept de «physique de la turbulence» est fonction de l'échelle à laquelle on se place.

Pour le puriste, l'une et l'autre approche n'ont connu que des succès limités : les structures organisées identifiées jusqu'ici diffèrent en effet d'une classe d'écoulements à une autre, comme diffèrent les "constantes" permettant de modéliser au mieux le comportement moyen du milieu turbulent à une certaine échelle d'observation. Il n'y a guère que pour les plus petites échelles d'une turbulence à grand nombre de Reynolds, bien développée et éloignée de toute paroi, que l'on pourrait parler d'une physique spécifique et générale de la turbulence, à condition toutefois de faire abstraction du rôle des grosses structures dans l'organisation des zones dissipatives.

Pour l'ingénieur, dont les critères d'appréciation sont différents, la conclusion est heureusement beaucoup plus positive. On possède en effet aujourd'hui une description phénoménologique relativement précise d'un grand nombre de classes d'écoulements turbulents, et une compréhension assez satisfaisante de leur dynamique interne. Les modélisations physiques du comportement de la turbulence, à la base des "fermetures" des équations dans l'espace physique ou dans l'espace spectral, permettent par ailleurs dans la majorité des cas une description suffisamment précise de ses effets. En fonction du degré d'approximation qu'il recherche, et à condition qu'il puisse mettre en œuvre des moyens de calcul convenables, on peut donc dire que l'ingénieur dispose aujourd'hui d'une "Physique de la turbulence" relativement bien adaptée à ses besoins. 


\section{Références}

Agard. - Conf. Proceedings, $N^{\prime \prime} 386$, Unsteady aerodynamics 644 p., Paris, 1985.

ANdre J.C., Barrere M. - Turbulence fluide, Cours d'option C2, Ecole Polytechnique, 256 pages, 1981.

Anselmet F., Gagne Y., Hopfinger E.J., Antonia R.A. J. Fluid Mech., 140, pp, 63-89, 1984

ANTONIA R.A., ANSELmet F, and Chambers A.J. - J. Fluid Mech., 163, pp. 365-391, 1986.

Arzoumanian E, Leborgne M., Fulachier L. - Proceedings, 3rd Symp. on Turbulent Shear Flows, Davis, California, pp. 2.13-2.18, 1981.

Aurry N., Holmes P., Lumley J.L., Stone E. - Submitted to $J$ Fluid Mech., 1986

Berge P., Pomeau Y. - La Recherche, II. pp. 422-432. Avril 1980

Berge P., Pomeau Y., Vidal. C. - Loordre dans le chaos. Hermann, Paris, 1984.

Blackwelder R.F. and Eckelmann H. - J. Fluid Mech., 94. pp. 577-594, 1979.

Bradshaw P. - Ed.. Turbulence, 2" édition, Springer Verlag. Berlin, 1978

BRodKey R.S. - Turbulence in mixing operations, Acad. Press. Inc, New-York, 1975.

Cantwell B.J. - Amn. Rer, Fluid Mech., 13, Ann. Rev. Inc., Palo Alto, 457-515, 1981.

CARR L.W. - IUTAM, Toulouse, Unsteady nurbulen shear flows, R. Michel and al Ed., Springer Verlag, mai 1981.

CEBECI T., STRAITH A.M. - Analysis of turbulent boumdary layers. Acad. Press, New-York, 404 p., 1974

CHAPMAN D.R. - AIAA Joumal, 17, pp. 1293-1314, 1979.

CHOLLET J.P. - NCAR, Int. Report 0 901-82-12, 1982.

Coustelx J. - Ann. Rel. Fluid Mech., 18, pp. 173-196, 1986.

Dubois M., Atten P., Berge P. - La Recherche, 18, pp. 190-201, 1987.

Falco R.E. - The phisics of fluids, 20, Part II, pp. S124-S132, 1977

Gibson C.H., Friehe C.A., Mc Connell S.O. - The Physics of Fluids, 20, supplement, pp. S156-S167, 1977.

Guckenhemer J. - Ann. Res. Fluid Mech.. 18. pp. 15-31, 1986. Herbert T. - AGARD. Conf. Proceedings. N" 224, 1977.

Hussain A.K. - The Phisics of Fluids, 26, pp. 2816-2850, 1983.

HUSSAIN A.K. - IUTAM S1mp. on Turbulence and chaotic phenomena in fluids, Kyoto, Japon, Septembre 1983.
LANFord O.E. - Amm. Rev: Fluid Mech., 14, pp. 347-364, 1982.

LESIEUR M. - Turbulence homogẻne el isolrope aspects phénoménologiques et statistiques, Cours ENSHG, Grenoble. 102 p. 1978.

Lesieur M. - J. Mec. Theor. et Appl., N" spécial. pp. 5-20, 1983

LibBY P.A., Williams F.A. - Editors, Turbulent reacting flous Springer Verlag, Berlin, 1980.

LUMLEY J.L. - Two-phase and non-newronian flows - in Tur bulence, P. Bradshaw Editor, Springer Verlig. pp. 289-324 1978.

LUMLEY J.L. - Colloque Euromech. "Transition and Turthlence ". Springer Verlag, pp. 215-242, 1981.

Kl.ine S.J., Reynoldos W.C., Schraeb F.A. and Rlistadllz P.W. - J. Fluid Mech., 30, pp. 741-773, 1967

Ku.INe S.J., Morkovin M.V., SOVran G., COCKrHLl. D.J. Proceedings Computation of urbulent boundary larers. 1968 AFOSR-IFP-Stanford Conference, 1969.

KLINE S.J., CANTWELL B.J., LILley G.M. - The 198(-81 AFOSRHTTM-Stanford Conference on complex turbulent flows. Stanford Univ.. California, 1981.

Mestayer P. - Thèse de Doct. ès Sc. Univ. Aix-Marseille II Sept. 1980

Mestayer P. - J. Fluid Mech., 125. pp. 475-503, 1982

Metais O. - Proceed. 5th Simp. on Turbulem Shear Flows. 22.27-22.32, 1985.

Michel. R., Cousteix J., Houdeville R. Ed., - Unsleddy nubulent shear flow's, Symposium IUTAM. Springer Verlag, 1981.

Paterson R.W. and Abernathy F.H. -- J. Fluid Mech., 51, pp. 177.185. 1972.

Rebuffet P. - Aérodinamique expérimentale, Librairie Polytech nique Ch. Béranger, Paris, 1950.

Reshotko L. - Ann. Rev. Fluid Mech., 8, pp. 311-349, 1976.

Swith F.T. - Ann. Rev. Fluid Mech., 18. pp. 197-220, 1986.

SOMMERIA J. - J.M.T.A., Numéro spécial, pp. 169-190. 1983.

Swinner H.L., Goleub J.P. - Editors, Hydrodynamic instabilities and the transition to turbulence, Springer Verlag, 292 p.. 1981.

Tavoularis S., Corrsin S. - J. Flitid Mech.. 104, 31 1-347 et 349-367, 1981

TLRBULENT SHEAR Flows 1 (1979) à V (1986), selected papers from the Intemational Symp. on Turbulem shear flows. Springer Verlag. Berlin. 1979 à 1986.

Wallace J.M. - in Springer Verlag, 235, Meier G.E.A. et Obermeier F. Eds, 1985. 


\section{Origine des figures}

Figure 1: Figure extraite de l'AIAA Journal, 17. N" 12, 1979, AIAA Special Publications, 1290 Avenue of the Americas, New-York, N.Y. 10019.

Figure 2 : Figure extraite de "Turbulence in Mixing Operations", Edited by R.S. Brodkey, Academic Press Inc., New-York, 1975.

Figure 3(a): Figure extraite de J. Fluid Mech., 5l, part 1, pp. 177-185, Cambridge University Press, 32 East 57th Street, New-York, N.Y. 10022.

Figure 3 (b): Figure extraite de "Aérodynamique expérimentale», Librairie polytechnique $\mathrm{Ch}$. Béranger, 15, rue des Saints-Pères, Paris, Edition 1950.

Figure 4: Figure extraite de "La Recherche », $11, \mathrm{~N}$ " 110, p. 431 , Avril 1980

Figure 6: Figure extraite de AGARD Conference Proceedings, N" 224, AGARD, 7, rue Ancelle, 92200 Neuilly sur Seine.

Figure 8(a): Figure extraite de : J. Fluid Mech., 30, part 4, p. 770, Cambridge University Press, 32 East 57th Street, New-York, N.Y. 10022.

Figure 8(b): Figure extraite de : J. Fluid Mech., 94, part 3, p. 589 , Cambridge University Press, 32 East 57 th Street. New-York, N.Y. 10022.

Figure 8(c): Figure extraite de : Lecture Notes in Physics, p. 256, 235, Meir G.E.A., Obermeier F., Eds, Springer-Verlag, Berlin, Heidelberg, Germany.
Figure 9: Figure extraite de: Proceedings of an International Symposium IUTAM, Washington, Juin 1976. The Physics of Fluids, 20, $\mathrm{N}^{\circ}$ 10, Part II, Octobre 1977, published by American Institute of Physics, New-York, 1977.

Figure 10: Figure extraite de :IUTAM Symposium on Turbulence and Chaotic Phenomena in Fluids, September 5-10, 1983. Kyoto, Japan, Elsevier Science Publishers, B.V., P.O. Box 1991, 1000 BZ, Amsterdam, Nederland (1984).

Figure 11 (Haut): Figure extraite du Bullet in de la Direction des Etudes et Recherches (E.D.F.) No 4, Série A, 198I, p. 255, Editeur : RGE SA, 48, rue de la Procession, 75724 Paris Cedex 15.

Figure 1/ (Bas): Figure extraite de : J. Fluid Mech., 104. p. 355, 1981, Cambridge University Press, 32 East 57th Street, New-York, N.Y. 10022.

Figure 12(a): Figure extraite de: J. Fluid Mech., 163, p. 382 (1986) Cambridge University Press, 32 East 57 th Street, New-York, N.Y. 10022

Figure 12(b): Figure extraite de : J. Fluid Mech., 104. p. 355 (1981) Cambridge University Press, 32 East 57th Street, New-York, N.Y. 10022.

Figure 13: Figure extraite de : J. Fluid Mech., 163, p. 387 (1986) Cambridge University Press, 32 East 57 th Street, New-York, N.Y. 10022.

Figure 14 : Figures extraites de : I.U.T.A.M. Symposium Toulouse, France, 5-8 mai 1981, published by Springer-Verlag, Berlin, Heidelberg, 1981, Germany.

Figure 15 : Figure extraite de : J.M.T.A., Numéro spécial, p. 178 (1983) Gauthier-Villars Editeur, 70, rue de St Mandé, 93100 Montreuil.

Adresse de l'auteur

Monsieur M. Coantic

Directeur de l'Institut de mécanique

statistique de la turbulence

12, avenue du Général Leclerc

13000 Marseille 